\title{
Nelson-Barr relaxion
}

\author{
Oz Davidi, ${ }^{1}$ Rick S. Gupta, ${ }^{1,2}$ Gilad Perez, ${ }^{1}$ Diego Redigolo, ${ }^{1,3}$ and Aviv Shalit ${ }^{1}$ \\ ${ }^{1}$ Department of Particle Physics and Astrophysics, Weizmann Institute of Science, Rehovot 7610001, Israel \\ ${ }^{2}$ Institute for Particle Physics Phenomenology, Department of Physics, \\ Durham University, DH1 3LE, Durham, United Kingdom \\ ${ }^{3}$ Raymond and Beverly Sackler School of Physics and Astronomy, \\ Tel-Aviv University, Tel-Aviv 69978, Israel
}

(Received 19 March 2018; revised manuscript received 4 July 2018; published 12 February 2019)

\begin{abstract}
Cosmological relaxation models in which the relaxion is identified with the QCD axion, generically fail to account for the smallness of the strong $C P$ phase. We present a simple alternative solution to this "relaxion $C P$ problem" based on the Nelson-Barr mechanism. We take $C P$ to be a symmetry of the UV theory, and the relaxion to have no anomalous coupling with QCD. The nonzero vacuum expectation value of the relaxion breaks $C P$ spontaneously, and the resulting phase is mapped to the Cabibbo-KobayashiMaskawa phase of the Standard Model. The extended Nelson-Barr quark sector generates the relaxion "rolling" potential radiatively, relating the new physics scale with the relaxion decay constant. With no new states within the reach of the LHC, our relaxion can still be probed in a variety of astrophysical and cosmological processes, as well as in flavor experiments.
\end{abstract}

DOI: $10.1103 /$ PhysRevD.99.035014

\section{INTRODUCTION}

The large hierarchy between the electroweak (EW) scale and the Planck scale, and the smallness of the strong $C P$ phase compared to the Cabibbo-Kobayashi-Maskawa (CKM) phase are two of the main mysteries of modern particle physics. In this paper, we solve the two problems in a unified way by combining cosmological relaxation [1] with the Nelson-Barr (NB) mechanism [2-4].

The key feature of cosmological relaxation is to make the Higgs mass dependent on the cosmological evolution of the relaxion. The relaxion is the pseudo-NambuGoldstone boson of a spontaneously broken Abelian symmetry $U(1)_{\text {clock }}$ that gets explicitly broken by two sequestered sectors with exponentially hierarchical charges. These sectors feed into the relaxion potential, generating exponentially different periodicities. After the relaxion dynamics is resolved, the ratio of the two periodicities is related to the hierarchy between the EW scale and the UV cutoff, where extra new physics stabilizing the Higgs mass is expected.

The structure of the relaxion potential forces it to get a vacuum expectation value (VEV), introducing a $C P$ violating phase in the theory $[1,5]$. This property causes the

Published by the American Physical Society under the terms of the Creative Commons Attribution 4.0 International license. Further distribution of this work must maintain attribution to the author(s) and the published article's title, journal citation, and DOI. Funded by SCOAP ${ }^{3}$. "relaxion $C P$ problem": the difficulty of identifying the relaxion with the QCD axion, and connecting the strong $C P$ problem with the naturalness of the EW scale. In this paper, we show that the relaxion $C P$ problem turns into a blessing if the large periodicity of the relaxion potential is generated via an NB model, such as the ones described in Refs. [6,7].

Following the NB basic setup, we assume that the UV theory preserves $C P$, and that the $U(1)_{\text {clock }}$ has zero QCD anomaly. A discrete symmetry ensures that the $C P$-violating phase, controlled by the relaxion VEV, is mapped into the CKM phase, while the strong $C P$ phase remains zero at tree level. In order to keep the strong $C P$ phase below the bounds from measurements of the neutron dipole moment [8], the NB sector should be feebly coupled to the source of $C P$ violation, so that radiative corrections are under control [7].

The NB relaxion solves the strong $C P$ problem together with the hierarchy problem by means of the dynamics of a single light degree of freedom. This should be contrasted with more conventional solutions of the hierarchy problem, like supersymmetry or compositeness, where a tension with $C P$ observables is often present (see e.g., Refs. [9-12]), and the strong $C P$ problem is typically addressed by adding new degrees of freedom disconnected from the Higgs sector.

Once the NB sector is specified, the relaxion potential with large periodicity is computable, and the UV cutoff gets connected to the relaxion decay constant. This provides a unique interplay between the LHC phenomenology, bounding the UV cutoff from below, and the low-energy phenomenology of the relaxion itself. We show how the LHC bounds together with a successful solution of the strong $C P$ problem imply that the spontaneous breaking of 
$U(1)_{\text {clock }}$ should happen at a high scale. As a consequence, the NB relaxion phenomenology is generically pretty elusive, even though a non-negligible relaxion-Higgs mixing can be probed in astrophysical and cosmological processes, and in flavor factories [5].

At the end of this paper, we discuss how the spontaneous breaking of $U(1)_{\text {clock }}$ at a high scale poses a "relaxion quality problem," which is related to the theoretical challenge of screening Planck-suppressed effects in order to preserve the peculiar structure of the relaxion potential.

\section{THE RELAXION AND ITS CP PROBLEM}

We first review the relaxion mechanism. The relaxion potential gets contributions from two different sectors: the "rolling" and the "backreaction"

$$
\begin{aligned}
V_{\text {roll }} & =\mu^{2}(\phi) H^{\dagger} H+\lambda_{H}\left(H^{\dagger} H\right)^{2}-r_{\text {roll }}^{2} \Lambda_{H}^{4} \cos \frac{\phi}{F}, \\
V_{\mathrm{br}} & =-M_{\mathrm{br}}^{2} H^{\dagger} H \cos \frac{\phi}{f}-r_{\mathrm{br}}^{2} M_{\mathrm{br}}^{4} \cos \frac{\phi}{f},
\end{aligned}
$$

where $H$ is the Standard Model (SM) Higgs doublet, $\phi$ is the relaxion, and we defined $\mu^{2}(\phi)=\kappa \Lambda_{H}^{2}-\Lambda_{H}^{2} \cos \frac{\phi}{F}$. $F(f)$ is the "large" ("small") periodicity of the rolling (backreaction) sector, $\Lambda_{H}$ is the UV cutoff of the Higgs effective theory, and $M_{\mathrm{br}}$ parametrizes the mass threshold controlling the backreaction potential. We also introduced the coefficient $\kappa \lesssim 1$ in front of the UV threshold to the Higgs mass, and the coefficients $r_{\text {roll }}, r_{\text {br }}$ which account for the possible differences between the Higgs-dependent and Higgs-independent contributions in the relaxion potential. Both coefficients satisfy $r_{\text {roll }}, r_{\mathrm{br}} \gtrsim 1 / 4 \pi$. The lower bound on $r_{\text {roll }}$ can easily be seen by closing the Higgs loop in the Higgs-dependent contributions in Eq. (1), while we refer to Refs. [5,13,14] for a discussion on how an analogous bound is obtained for the backreaction sector.

During inflation, the relaxion rolls down the potential from some initial value $\phi \lesssim \phi_{c} \approx-\left|F \cos ^{-1} \kappa\right|$, such that $\mu^{2}(\phi)>0$. While rolling down, the relaxion dissipates energy through Hubble friction, and when $\phi \gtrsim \phi_{c}, \mu^{2}(\phi)<0$ and the EW symmetry is broken.

In the EW broken phase, the backreaction potential $V_{\mathrm{br}}$ generates wiggles, allowing the relaxion to stop where the Higgs mass is at its measured value (see Ref. [1] for further details). This requirement gives an upper bound on the amplitude of the Higgs-independent contribution to the backreaction potential, which can be translated into an upper bound on the backreaction scale

$$
M_{\mathrm{br}} \lesssim \frac{v}{r_{\mathrm{br}}} \lesssim 4 \pi v .
$$

If the above condition is fulfilled, the relaxion stops at $\phi_{0}=\langle\phi\rangle$, where $\left|\mu\left(\phi_{0}\right)\right|=m_{h}$, and one gets

$$
\begin{gathered}
\sin \frac{\phi_{0}}{f} \sim \sin \frac{\phi_{0}}{F} \sim \mathcal{O}(1), \\
\partial_{\phi} V=0 \Rightarrow \frac{\Lambda_{H}}{\Lambda_{\mathrm{br}}} \sim\left(\frac{F}{r_{\text {roll }}^{2} f}\right)^{1 / 4},
\end{gathered}
$$

where $\Lambda_{\mathrm{br}}=\sqrt{M_{\mathrm{br}} v}$ is the backreaction scale. Equation (5) shows that a large ratio between the Higgs bare mass $\Lambda_{H}$ and $\Lambda_{\mathrm{br}}$ can be achieved from a large ratio of the periodicities $F / f$.

The clockwork mechanism [15-17] gives a calculable example where $F / f \gg 1$. This construction introduces $N+1$ spontaneously broken Abelian symmetries at different sites of a moose diagram. The different sites are connected by $\epsilon$-suppressed operators, breaking explicitly $N$ of the Abelian symmetries. The clockwork potential reads

$$
\begin{aligned}
V_{\text {clock }} & =\sum_{j=0}^{N}-m^{2}\left|\Phi_{j}\right|^{2}+g_{\text {clock }}^{2}\left|\Phi_{j}\right|^{4}+\Delta V_{\text {clock }}, \\
\Delta V_{\text {clock }} & =-\sum_{j=0}^{N-1}\left[\epsilon \Phi_{j}^{\dagger} \Phi_{j+1}^{3}+\text { H.c. }\right],
\end{aligned}
$$

where the symmetry-breaking scale is $f=m / g_{\text {clock }}$. By taking $|\epsilon| \ll g_{\text {clock }}^{2} \sim 1$, the radial modes can be decoupled, and we can write an effective action for the angular modes $\pi_{j}$ by setting $\Phi_{j}=\frac{f}{\sqrt{2}} e^{i \pi_{j} / f}$. The $\epsilon$-suppressed operators induce masses $m_{j}^{2} \approx \epsilon f^{2}$ for $N$ angular modes, stabilizing their VEVs at the origin. At the bottom of the spectrum, we are left with a single Nambu-Goldstone boson $\phi$, which nonlinearly realizes the $U(1)_{\text {clock }}$ symmetry: $\pi_{j} \rightarrow \pi_{j}+\frac{1}{3^{j}} f \alpha, \phi \rightarrow \phi+f \alpha$.

Since the overlap of $\phi$ with the site $j$ is suppressed by $\left\langle\pi_{j} \mid \phi\right\rangle \approx 1 / 3^{j}$, introducing an explicit breaking of $U(1)_{\text {clock }}$ at the site $j$ generates a potential for $\phi$ with periodicity of order $3^{j} f$. The desired hierarchy between the periodicities of the relaxion potential is then achieved by putting the backreaction sector at the zeroth site, and the rolling sector at the $N$ th site. All in all, we get $F / f \approx 3^{N}$.

As shown in Ref. [1], there are constraints on the Hubble parameter arising from, 1) the condition that classical rolling of the relaxion dominates over quantum fluctuations and 2) the requirement that the relaxion vacuum energy density is small compared to the total vacuum energy density. Together these conditions lead to a bound on the UV cutoff scale [18]

$$
\begin{aligned}
\Lambda_{H} & \lesssim\left(\frac{M_{\mathrm{Pl}}}{r_{\mathrm{roll}}}\right)^{1 / 2} \cdot\left(\frac{\Lambda_{\mathrm{br}}^{4}}{f}\right)^{1 / 6} \\
& \lesssim 10^{9} \mathrm{GeV} \cdot\left(\frac{1 / 4 \pi}{r_{\text {roll }}}\right)^{1 / 2} \cdot\left(\frac{10^{9} \mathrm{GeV}}{f}\right)^{1 / 6} \cdot\left(\frac{\Lambda_{\mathrm{br}}}{m_{h}}\right)^{2 / 3},
\end{aligned}
$$


where to obtain the second inequality, we saturated both the upper bound on $\Lambda_{\mathrm{br}}$ and the lower bound on $r_{\text {roll }}$.

Equation (4) is at the origin of the relaxion $C P$ problem. Having $\left|\mu\left(\phi_{0}\right)\right|=m_{h}$ at the stopping point implies $\phi_{0} \approx F$. Therefore, a phase $\theta_{N} \equiv \frac{\phi_{0}}{F} \sim \mathcal{O}(1)$ is induced at the $N$ th site of the clockwork. This gives also a phase $\theta_{0} \equiv \frac{\phi_{0}}{f} \sim$ $\mathcal{O}(1)$ at the zeroth site. Therefore, if the relaxion is identified with the QCD axion, and the backreaction potential is generated through an anomaly of $U(1)_{\text {clock }}$ with QCD, we have $\Lambda_{\mathrm{br}} \sim\left(v \Lambda_{\mathrm{QCD}}^{3}\right)^{1 / 4}$ and $\theta_{0}$ induces a QCD $\theta$ angle of $\mathcal{O}(1)$, which is experimentally excluded.

To generate $V_{\text {br }}$ via QCD, the relaxion dynamics should be modified in order to circumvent Eq. (4). This is the approach followed in Ref. [1], and more recently in Refs. [22,23]. A trivial solution of the relaxion $C P$ problem is instead to assume $U(1)_{\text {clock }}$ to be anomaly free with respect to QCD, and to generate the backreaction potential otherwise. However, the strong $C P$ problem would remain unaddressed, reducing the appeal of the original relaxion proposal [24].

We show here a third type of solution which assumes $C P$ to be a symmetry of the UV theory, and $U(1)_{\text {clock }}$ to be anomaly free. The rolling potential is generated by an NB sector like the one presented in Refs. [6,7], the phase $\theta_{N}$ is mapped into the CKM phase, and the strong $C P$ problem is solved without modifying the standard relaxion dynamics. As a small drawback, the backreaction sector becomes less minimal: it requires extra states below $4 \pi v$, and $r_{\mathrm{br}}$ in Eq. (2) to be small enough to suppress the Higgs-independent wiggles. We provide an example backreaction sector in the Appendix B (see also Refs. [1,13,14,25]).

\section{THE NELSON-BARR RELAXION}

We now present a simple implementation of the NB relaxion. The NB sector is borrowed from Ref. [6],

$$
\mathcal{L}_{\mathrm{NB}}=\left[y_{i}^{\psi} \Phi_{N}+\tilde{y}_{i}^{\psi} \Phi_{N}^{*}\right] \psi u_{i}^{c}+\mu \psi \psi^{c}+\text { H.c. },
$$

and all couplings are real in our basis. The SM up sector, $\mathcal{L}_{Y^{u}}=Y_{i j}^{u} H Q_{i} u_{j}^{c}+$ H.c., gets extended by two additional vector-like Weyl fermions, $\psi$ and $\psi^{c}$, in the fundamental and antifundamental of $S U(3)_{C}$, and with opposite hypercharges $\pm 2 / 3$. The single complex scalar that breaks $C P$ spontaneously in the NB model of Ref. [6] is here identified with $\Phi_{N}$ at the $N$ th site of the clockwork moose diagram. The $U(1)_{N}$ gets explicitly broken by the interactions of $\Phi_{N}$.

The structure of the renormalizable couplings of $\Phi_{N}$ in Eq. (9) is enforced by a $\mathbb{Z}_{2}$ symmetry under which $\Phi_{N}, \psi$, and $\psi^{c}$ are charged. This discrete symmetry forbids operators like $\Phi_{N} \psi \psi^{c}$ and $H Q \psi^{c}$, and is spontaneously broken by the VEV of $\Phi_{N}$.

In our minimal setup, the rolling potential for the relaxion $\phi$ is generated from Eq. (9). Matching to the potential in Eq. (1), we get
$\Lambda_{H} \sim \frac{\sqrt{y_{i}^{\psi} \tilde{y}_{j}^{\psi}\left(Y^{u^{\dagger}} Y^{u}\right)_{i j}}}{4 \pi} f, \quad r_{\text {roll }} \sim \frac{4 \pi g_{\text {clock }} \sqrt{y_{k}^{\psi} \tilde{y}_{k}^{\psi /}}}{y_{i}^{\psi /} \tilde{y}_{j}^{\psi}\left(Y^{u^{\dagger}} Y^{u}\right)_{i j}}$.

In the presence of the backreaction potential, the relaxion stops where $\theta_{N} \sim \mathcal{O}(1)$, as dictated by Eq. (4). Setting $\Phi_{N}$ to its VEV, we can define $B_{i}=\frac{f}{\sqrt{2}}\left(y_{i}^{\psi} e^{i \theta_{N}}+\tilde{y}_{i}^{\psi} e^{-i \theta_{N}}\right)$. The $4 \times 4$ mass matrix of the up quarks at tree level is

$$
M^{u}=\left(\begin{array}{cc}
(\mu)_{1 \times 1} & (B)_{1 \times 3} \\
(0)_{3 \times 1} & \left(v Y^{u}\right)_{3 \times 3}
\end{array}\right),
$$

so that, even though the above mass matrix is complex, we find $\bar{\theta}_{\mathrm{QCD}}=\operatorname{Arg}\left(\operatorname{det}\left(M^{d}\right)\right)+\operatorname{Arg}\left(\mu \cdot \operatorname{det}\left(v Y^{u}\right)\right)=0$. Integrating out the heavy quarks for $\mu^{2}+B_{i} B_{i}^{*} \gg v^{2}$, we find the effective $3 \times 3$ mass squared of the SM up quark sector:

$$
\left[M_{\mathrm{eff}}^{u} M_{\mathrm{eff}}^{u \dagger}\right]_{i j} \sim v^{2} Y_{i k}^{u} Y_{j k}^{u *}-\frac{v^{2} Y_{i k}^{u} B_{k}^{*} B_{\ell} Y_{j \ell}^{u *}}{\mu^{2}+|B|^{2}} .
$$

A phase in the unitary matrix $V_{u}^{L}$, diagonalizing the matrix in Eq. (12), would lead to a phase in the CKM matrix $V_{\mathrm{CKM}}=V_{u}^{L \dagger} V_{d}^{L}$. One can show that $\delta_{\mathrm{CKM}}$ vanishes in the limit $\left|\vec{y}^{\psi} \times \overrightarrow{\tilde{y}}^{\psi}\right| /\left|\vec{y}^{\psi}+\overrightarrow{\tilde{y}}^{\psi}\right|^{2} \ll 1$. To ensure $\delta_{\mathrm{CKM}} \sim \mathcal{O}(1)$, we take $y_{i}^{\psi} \sim \tilde{y}_{i}^{\psi} \sim y_{\psi}$ for all $i$, and $\mu<\left|B_{i}\right| \sim\left|y_{\psi}\right| f$. In the limit where $\mu \ll\left|B_{i}\right|$, one of the eigenvalues of the matrix in Eq. (12) is suppressed by $\mu^{2} /\left|B_{i}\right|^{2}$, and can possibly account for the hierarchy in quark masses. However, the resulting mass eigenstate should not be smaller than the up mass. We thus get a rough lower bound for $\mu$ : $\sqrt{m_{u} / v}|B| \leq \mu$. For concreteness, we assume below that $\mu \sim\left|B_{i}\right|$.

We next discuss the corrections to $\bar{\theta}_{\mathrm{QCD}}$ beyond the treelevel approximation discussed. The leading corrections were classified in Ref. [7], and are coming from either radiative corrections or from higher-dimension operators. A detailed analysis of the different contributions can be found in the Appendix. The experimental bound on $\bar{\theta}_{\mathrm{QCD}}$ translates into an upper bound on $y_{i}^{\psi}$ and $\tilde{y}_{i}^{\psi}$ :

$$
\Delta \bar{\theta}_{\mathrm{QCD}}^{\mathrm{UV}} \lesssim 10^{-10} \Rightarrow\left|y_{i}^{\psi}\right| \sim\left|\tilde{y}_{i}^{\psi}\right| \lesssim 10^{-2}, 10^{-4} .
$$

The two bounds above depend on the flavor structure assumed: the weaker [stronger] applies to models with minimal flavor violation (MFV) [quasidiagonal] structure. In MFV-like models, flavor violation arises from the SM Yukawas or $y_{i}^{\psi}, \tilde{y}_{i}^{\psi \prime}$ (see e.g., Refs. [26,27]).

Note that, in addition to the contributions to the strong $C P$ and CKM phases at the renormalizable level, integrating out the vector-like pair will also generate dimension-six $C P$-violating operators that contribute, for instance, to electric or chromoelectric dipole moments. These operators 
will however be suppressed by the mass of the fermion, which is larger than the $\mathcal{O}(\mathrm{TeV})$ bound on the scale of such operators [28].

In our construction, the cutoff of the Higgs sector is much smaller than the one of the clockwork theory, $\Lambda_{H} \ll \Lambda_{f}$. However, our analysis does not include higher-dimensional operators arising at the IR threshold $\Lambda_{H}$. A proper estimation of these effects would require a concrete UV completion of the NB relaxion, embedding the Higgs IR threshold, $\Lambda_{H}$, in a full model of supersymmetry (SUSY) or compositeness. These UV completions are challenging, and beyond the scope of this work (see Refs. [29-31] for attempts at relaxion UV completions, and also Refs. [7,32,33] for SUSY or composite UV complete NB models).

Within our simple model, the radiative contributions to $\Delta \bar{\theta}_{\mathrm{QCD}}$ of the new states at $\Lambda_{H}$ can be subleading with respect to the ones considered here if the interactions with the clockwork chain are suppressed enough. A better solution would be to modify the NB construction by softening the breaking of $U(1)_{\text {clock }}$ (see e.g., Ref. [13]). With such a construction, one could possibly achieve $\Lambda_{H} \sim \Lambda_{f}$, suppressing the higher-dimensional operators at $\Lambda_{H}$ as much as the ones we considered here. We leave this matter for future investigations, and focus here on the simplest possible model, where we introduce an explicit breaking of the $U(1)_{N}$, like in Eq. (9), which leads to the "quadratically divergent" rolling potential of Eq. (10).

Finally, let us mention that despite the fact that $C P$ is a good symmetry in our Lagrangian, the $\mathcal{O}(1)$ spontaneous $C P$ violation in Eq. (9) and the fact that relaxion breaks $C P T$ spontaneously while rolling can lead to successful baryogenesis models $[34,35]$.

\section{PHENOMENOLOGY}

We now assess the parameter space of the NB relaxion, and study its phenomenology. In our minimal setup, the rolling potential is generated from the NB sector at the $N$ th site. This implies that the cutoff $\Lambda_{H}$ is related to the scale $f$. Taking Eq. (10) at face value, given a lower bound on the new physics scale $\Lambda_{H} \gtrsim 5 \mathrm{TeV}$, and the upper bound on the explicit breaking of the $U(1)_{N}$ in Eq. (13), we get

$f^{\min }=\left(10^{7}, 10^{9}\right) \mathrm{GeV} \cdot\left(\frac{\Lambda_{H}}{5 \mathrm{TeV}}\right) \cdot \frac{\left(10^{-2}, 10^{-4}\right)}{\sqrt{y^{\psi /} \tilde{y}^{\psi}}}$,

where the weaker (stronger) lower bound is related to models with MFV (quasidiagonal) flavor structure. From now on, we focus on the MFV parameter space, presented in Fig. 1, and leave the discussion on the quasidiagonal case for the Appendix A.

The mass of the relaxion is controlled by the backreaction scale $m_{\phi} \approx \Lambda_{\mathrm{br}}^{2} / f$, so that the upper bound in Eq. (3) gives an upper bound on the relaxion mass:

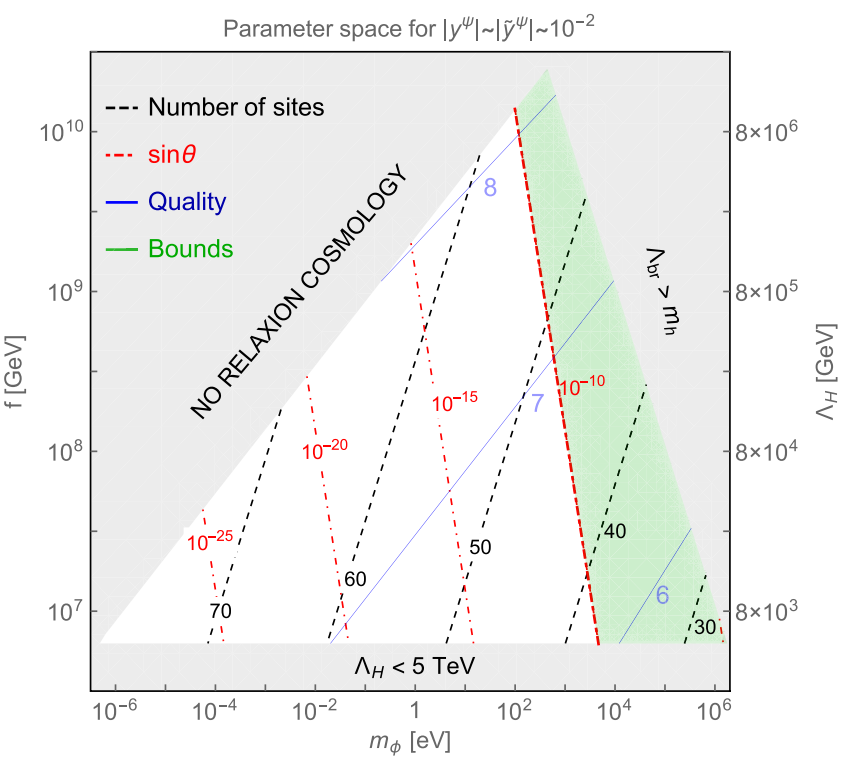

FIG. 1. The white region shows the allowed parameter space of the Nelson-Barr relaxion. The contours indicate the number of clockwork sites (black dashed), the relaxion-Higgs mixing (red dot-dashed), and the minimal dimension of the Planck-suppressed operators that should be forbidden (thin blue). The green-shaded region combines constraints from astrophysics [36-39], extragalactic background light [5], and flavor-violating kaon decays [40]. The upper left grey region is ruled out by the bound in Eq. (8).

$m_{\phi}^{\max }=20 \mathrm{MeV} \cdot \frac{5 \mathrm{TeV}}{\Lambda_{H}} \cdot \frac{\sqrt{y^{\psi} \tilde{y}^{\psi}}}{10^{-2}}$. The constraint in Eq. (8), coming from a successful relaxion cosmology, gives instead a lower bound on the relaxion mass: $m_{\phi}^{\min }=$ $2 \times 10^{-6} \mathrm{eV} \cdot\left(\frac{\Lambda_{H}}{5 \mathrm{TeV}}\right)^{5 / 2} \cdot \frac{10^{-2}}{\sqrt{y^{\psi} \tilde{y}^{\Psi}}}$. The three constraints above explain the boundaries of the allowed triangle in Fig. 1. The maximal allowed decay constant $f^{\max }=3 \times 10^{10} \mathrm{GeV}$. $\left(\frac{10^{-2}}{\sqrt{y^{4} \tilde{y}^{\tilde{W}}}}\right)^{3 / 7}$ is achieved for $m_{\phi}^{\min }=m_{\phi}^{\max }$. The available parameter space shrinks for smaller $\sqrt{y^{\psi /} \tilde{y}^{/ /}}$until $f^{\max } \approx f^{\text {min }}$. This happens for $\sqrt{y^{\psi /} \tilde{y}^{\psi /}} \sim 10^{-9}$, which can be taken as the minimal amount of $U(1)_{N}$ breaking in our setup.

Through the backreaction sector, a relaxion-Higgs mixing is generated. The mixing angle, $\sin \theta \approx 7 \times 10^{-7} \frac{f}{\mathrm{GeV}} \cdot \frac{m_{\phi}^{2}}{\mathrm{GeV}^{2}}$, is plotted in Fig. 1 where the exclusion band for $\sin \theta \gtrsim 10^{-10}$ accounts for astrophysical probes [36-39], and flavor experiments [40]. More details are given in the Appendix C.

Another interesting feature of our setup is the changing of $\delta_{\mathrm{CKM}} \approx\langle\phi\rangle / F$ during the relaxion rolling. However, this would hardly lead to observable effects, unless the flavor suppression of the SM CP violation is somehow reduced [41].

\section{QUALITY PROBLEMS}

The spontaneous breaking of the global $U(1)_{\text {clock }}$ at a high scale introduces a "relaxion quality problem," which shares some similarities with the "axion quality problem" 
discussed in Ref. [42]. The basic issue is that gravity is not expected to respect any global symmetry, as suggested by many theoretical arguments [43-47]. As a consequence, gravity-induced higher-dimensional operators would generically break the $U(1)_{\text {clock}}$, generating a potential for the relaxion. The most dangerous gravity contributions to the potential are the ones controlled by operators like $\frac{\Phi_{0}^{4+\Delta}}{M_{\mathrm{Pl}}^{\Delta}}$, with $\Delta \geq 1$ (see Ref. [48] for an early discussion on the quality problem of the clockwork construction). These operators generate $\Delta V_{\text {grav }} \sim \frac{f^{4+\Delta}}{M_{\mathrm{P}}^{\Delta}} \cos \frac{\Delta \phi}{f}$, which, being independent of the Higgs VEV, should be smaller than the backreaction potential in Eq. (2) in order for the relaxion mechanism to work. [49] By imposing $\Delta V_{\text {grav }} \lesssim$ $V_{\text {br }}$ together with Eq. (3) and Eq. (14), we get $\Delta \gtrsim$ $\left[\frac{4 \log \frac{\Lambda_{b \mathrm{r}}}{f}}{\log \frac{f}{M_{\mathrm{Pl}}}}\right] \approx 2$, which implies that gravity-induced operators up to dimension six should be forbidden. This is a generic problem of every relaxion model with a high decay constant $f$. Since imposing gauged $\mathbb{Z}_{N}$ symmetries on the gravity theory seems challenging in the clockwork setup, the only way of addressing this problem would be to build a UV completion where the $U(1)_{\text {clock }}$ is an accidental symmetry emerging in the infrared. This last possibility has been explored in the context of clockwork constructions in Refs. [17,51].

The NB sector can also be affected by gravity-induced higher-dimensional operators. These operators evade our power counting because they are not controlled by powers of $y_{\psi}$, and they break MFV, leading to dangerous contributions to $\bar{\theta}_{\mathrm{QCD}}$. If dimension-five operators are forbidden, the effects of dimension-six operators are already small enough for $f \sim 10^{9} \mathrm{GeV}$ to guarantee a successful NB mechanism.

Raising $f$ would exacerbate the problem, making it necessary to embed the NB construction in a gauge theory where the $C P$-violating phase arises from a condensate (see e.g., Refs. $[33,52,53])$.

\section{CONCLUSIONS}

The main lesson of this work is that combining the relaxion mechanism with the NB construction leads to two positive outcomes: (i) the "relaxion $C P$ problem," induced by the $\mathcal{O}(1) C P$ phase of the relaxion vacuum expectation value, is solved; (ii) the relaxion $C P$ phase becomes the origin of the CKM phase. Our model serves as an existence proof of the NB relaxion setup, focusing on the simplest possible implementation, which captures some generic features of the construction. We showed how the NB sector provides the relaxion "rolling" potential, connecting the relaxion decay constant with the cutoff scale, where new physics stabilizing the Higgs mass is expected. Within our setup, the cosmological evolution of the relaxion is kept as minimal as in the original proposal [1]. This should be contrasted with other proposals to solve the relaxion $C P$ problem, which either require the relaxion potential to be modified after inflation [1], or the classical evolution of the relaxion to be overcome by its large quantum fluctuations [22]. A feeble coupling between the NB sector and the relaxion seems necessary in order to guarantee the success of the construction. This makes the relaxion detection challenging, even though signatures are expected for maximal backreaction scales in astrophysical, cosmological and flavor observables. Breaking the $U(1)_{\text {clock }}$ at a high scale introduces the challenge of protecting the entire construction from Planck-suppressed operators. It would be interesting to see if any of these problems can be ameliorated in more elaborate versions of the NB relaxion, which include supersymmetry or compositeness to stabilize the UV cutoff (see Refs. [7,33] for a discussion on the NB mechanism in these frameworks). The NB construction is deeply connected to the SM flavor structure. It is then interesting to embed the NB relaxion in a full model of flavor à la Froggatt-Nielsen [54]; this was presented in Ref. [25].

\section{ACKNOWLEDGMENTS}

We thank David Kaplan, Zohar Komargodski, Yossi Nir, Lorenzo Ubaldi, Tomer Volansky, and Jure Zupan for useful discussions. We also thank Nathaniel Craig and Claudia Frugiuele for comments on the draft, and Alfredo Urbano and Michael Dine for fruitful discussions on the quality problem. The work of G. P. is supported by grants from the United States-Israel Binational Science Foundation (BSF), European Research Council (ERC), Israeli Science Foundation (ISF), Minerva, and the Weizmann-UK Making Connections Program.

\section{APPENDIX A: RADIATIVE STABILITY OF NELSON-BARR}

We comment here on the radiative stability of the NB construction we presented. Dangerous radiative corrections can be parametrized in a shift of the mass matrix of the up sector, which we schematically write as $M^{u} \rightarrow M^{u}+\Delta M^{u}$, where $\Delta M^{u} \ll M^{u}$ is

$$
\Delta M^{u} \equiv\left(\begin{array}{cc}
(\delta \mu)_{1 \times 1} & (\delta B)_{1 \times 3} \\
\left(v \delta Y^{\psi^{c}}\right)_{3 \times 1} & \left(v \delta Y^{u}\right)_{3 \times 3}
\end{array}\right),
$$

and its contribution to $\bar{\theta}_{\mathrm{QCD}}$ reads

$$
\Delta \bar{\theta}_{\mathrm{QCD}} \approx \operatorname{Im}\left[\operatorname{Tr}\left(\left(M^{u}\right)^{-1} \Delta M^{u}\right)\right] .
$$

There are three different types of contributions:

$$
\begin{aligned}
& \delta \bar{\theta}_{1}=\operatorname{Im}\left[\mu^{-1} \delta \mu\right], \\
& \delta \bar{\theta}_{2}=-\operatorname{Im}\left\{\operatorname{Tr}\left[\mu^{-1} B\left(Y^{u}\right)^{-1} \delta Y^{\psi^{c}}\right]\right\}, \\
& \delta \bar{\theta}_{3}=\operatorname{Im}\left\{\operatorname{Tr}\left[\left(Y^{u}\right)^{-1} \delta Y^{u}\right]\right\} .
\end{aligned}
$$


TABLE I. Charges of the spontaneously broken $\mathbb{Z}_{2}$ and the spurion charge assignment for the three broken $U(1)$ symmetries controlling the radiative corrections.

\begin{tabular}{lcrcc}
\hline \hline & $\mathbb{Z}_{2}$ & $U(1)_{N}$ & $U(1)_{\psi}$ & $U(1)_{\mu}$ \\
\hline$\Phi_{N}$ & - & -1 & 0 & 0 \\
$\psi$ & - & 0 & 1 & 0 \\
$\psi^{c}$ & - & 0 & -1 & 1 \\
$y_{i}^{\psi}$ & + & 1 & -1 & 0 \\
$\tilde{y}_{i}^{\psi \prime}$ & + & -1 & -1 & 0 \\
$\mu$ & + & 0 & 0 & -1 \\
\hline \hline
\end{tabular}

By promoting the couplings $y_{i}^{\psi}, \tilde{y}_{j}^{\psi}$, and $\mu$ to spurions, one can see from Table I that there is a (set of) charge assignment(s) of three $U(1)$ 's, acting nontrivially on $\Phi_{N}$ and the vector-like fermions. By assuming that the full theory respects the same selection rules, we can parametrically estimate by spurion counting the leading-order contributions to $\bar{\theta}_{\mathrm{QCD}}$.

We first estimate the contributions from integrating out the clockwork scalar chain. Inspecting Table I, we conclude that the $\mathcal{O}\left(y^{\psi /} \tilde{y}^{\psi /}\right)$ contributions to Eq. (A3) should be proportional to $\operatorname{Im}\left[y_{i}^{\psi} \tilde{y}_{i}^{\psi * *}\left\langle\Phi_{N}\right\rangle^{2}+y_{i}^{\psi *} \tilde{y}_{i}^{\psi /}\left\langle\Phi_{N}^{*}\right\rangle^{2}\right]=0$, where we have used the fact that our Lagrangian is invariant under the interchange $\left(y_{i}^{\psi}, \Phi_{N}\right) \leftrightarrow\left(\tilde{y}_{i}^{\psi}, \Phi_{N}^{*}\right)$. The contributions to Eqs. (A4) and (A5) similarly vanish at the same order, and no misalignment can be produced to get a nonzero phase.

Our spurion analysis is in agreement with the explicit computation of Refs. [6,7], which shows that the first dangerous loop corrections in this model are controlled by Higgs-portal operators like $H^{\dagger} H \Phi_{N}^{2}$. These are generated at one loop in our setup, together with the rolling potential for the relaxion, only after integrating out the heavy quarks. As a consequence, the leading contributions to Eqs. (A3)-(A5) arise at two loops, and their sum scales as

$$
\Delta \bar{\theta}_{\mathrm{QCD}}^{\text {clock }} \sim \frac{\left(y^{\psi} \tilde{y}^{\psi}\right)^{2}}{256 \pi^{4}} \frac{\left|\left\langle\Phi_{N}\right\rangle\right|^{2}}{m^{2}} \sin \left(2 \theta_{N}\right),
$$

where $m$ is the mass of the radial mode in the clockwork construction. The upper bound on $\bar{\theta}_{\mathrm{QCD}}$ translates into an upper bound on the explicit breaking of the $U(1)_{N}$ :

$$
\Delta \bar{\theta}_{\mathrm{QCD}}^{\text {clock }} \lesssim 10^{-10} \Rightarrow\left|y^{\psi}\right| \sim\left|\tilde{y}^{\psi}\right| \lesssim 10^{-2} .
$$

We now consider the effects of higher-dimensional operators. The leading-order higher-dimensional operators generated at the cutoff scale of the clockwork theory, $\Lambda_{f} \sim 4 \pi f$, are

$$
\begin{aligned}
\mathcal{L}_{\mathrm{UV}} \supset & \frac{\tilde{y}_{j}^{\psi *}}{16 \pi^{2}}\left[y_{i}^{\psi} \alpha_{i j} \mu \frac{\Phi_{N}^{2}}{f^{2}} \psi \psi^{c}+\beta_{k j} \mu \frac{\Phi_{N}}{f^{2}} H Q_{k} \psi^{c}\right. \\
& \left.+y_{i}^{\psi} \gamma_{k \ell i j} \frac{\Phi_{N}^{2}}{f^{2}} H Q_{k} u_{\ell}^{c}\right]+ \text { H.c. },
\end{aligned}
$$

and other operators are obtained by interchanging $\left(y_{i}^{\psi}, \Phi_{N}\right) \leftrightarrow\left(\tilde{y}_{i}^{\psi}, \Phi_{N}^{*}\right)$. The first operator induces a contribution to $\bar{\theta}_{\mathrm{QCD}}$ which can be directly estimated through Eq. (A3)

$$
\Delta \bar{\theta}_{\mathrm{QCD}}^{\mathrm{UV}} \sim \frac{\alpha_{i j}}{16 \pi^{2}}\left(y_{i}^{\psi} \tilde{y}_{j}^{\psi}-y_{j}^{\psi} \tilde{y}_{i}^{\psi}\right) \frac{\left|\left\langle\Phi_{N}\right\rangle\right|^{2}}{f^{2}} \sin \left(2 \theta_{N}\right)
$$

and we again assumed the UV Lagrangian to be invariant under the interchange $\left(y_{i}^{\psi}, \Phi_{N}\right) \leftrightarrow\left(\tilde{y}_{i}^{\psi^{\prime}}, \Phi_{N}^{*}\right)$. In realizations of MFV [26], the coefficient $\alpha_{i j}$ is either proportional to the identity or to powers of $\left(Y^{u \dagger} Y^{u}\right)_{i j}$. In either case, the contribution to $\Delta \bar{\theta}_{\mathrm{QCD}}^{\mathrm{UV}}$ in Eq. (A9) vanishes. Higher powers in $\left(Y^{d \dagger} Y^{d}\right)_{i j}$ might lead to violations of this scaling [27,55], but will be highly suppressed. An analogous reasoning applies to the operators controlled by $\beta$ and $\gamma$, so that the contribution to $\Delta \bar{\theta}_{\mathrm{QCD}}^{\mathrm{UV}}$ at leading order in $y_{[i}^{\psi} \tilde{y}_{j]}^{\psi}$ identically vanishes. The first nonzero contribution is then of order $\mathcal{O}\left(y_{\psi}^{4}\right)$, and hence suppressed compared to the generic case as in Eq. (A7).

Notice that the other two operators in Eq. (A8) give contributions of the same order as the ones from Eq. (A9) only because the factors $\left(Y^{u}\right)^{-1} \beta$ and $\left(Y^{u}\right)^{-1} \gamma$, in Eqs. (A4) and (A5), do not lead to any enhancement in MFV scenarios. More generally, this enhancement is not present in a large class of flavor models where the couplings of the first two generations are suppressed, resulting in quasidiagonal textures. This is the case in various $U(2)$ models, in $U(1)$ horizontal models, and in models where the flavor puzzle is addressed through hierarchies in the anomalous dimensions (for a discussion, see e.g., Refs. [55-61] and references therein). In this class of models, the contributions of order $\mathcal{O}\left(y_{\psi}^{2}\right)$, such as the ones in Eq. (A9), do not vanish, resulting in the stronger bound $y_{\psi} \lesssim 10^{-4}$.

The clockwork intersite coupling in Eq. (7) of the main text, implies that all the scalar VEVs of the clockwork chain break the $\mathbb{Z}_{2}$ symmetry spontaneously. Additional contributions obtained by replacing, in Eq. (A8), $\Phi_{N} \rightarrow \Phi_{i}$ with $0<i<N$ do not, however, add new complex phases. Consequently, these do not change our power counting. There are possible new contributions that arise through mixing of the NB fields with the ones on the first site, where the backreaction sector lies. However, these are suppressed by powers of $\sim \epsilon^{N}$, and thus can be safely neglected.

Finally, there can be a model-dependent contribution to $\bar{\theta}_{\mathrm{QCD}}$ arising from the spontaneous $C P$ violation in the backreaction sector. This turns out to be harmless for small enough backreaction scales. An explicit example is discussed in the next section. 


\section{APPENDIX B: THE BACKREACTION SECTOR}

We complete the relaxion part of the construction by introducing an example of a working backreaction sector, which acts as a proof of concept for the NB relaxion idea. Here, we present a modified version of the familon model that was presented in Refs. [5,13], and we refer to Ref. [25], where another similar example was recently presented.

The backreaction Lagrangian consists of the following interaction terms:

$$
\begin{aligned}
\mathcal{L} \supset & -Y_{1} \frac{\Phi_{0}}{\Lambda_{\mathrm{FN}}} L H N-Y_{2} \frac{\Phi_{0}}{\Lambda_{\mathrm{FN}}} L^{c} H^{\dagger} N \\
& -m_{L} L L^{c}-m_{D} N N^{c}-\frac{m_{N^{c}}}{2} N^{c} N^{c}+\text { H.c. },
\end{aligned}
$$

where $\Phi_{0}$ is the clockwork scalar at the first site, $L$ has the same quantum numbers as the SM lepton doublet, $N$ is an SM singlet fermion, and $\left(L^{c}, N^{c}\right)$ are in the conjugate representation with respect to $(L, N) . \Lambda_{\mathrm{FN}}$ is a scale associated with the Froggatt-Nielsen [54] UV completion, and the backreaction sector is kept invariant under the $\mathbb{Z}_{2}$ transformation by taking $L$ and $L^{c}$ to be $\mathbb{Z}_{2}$ odd. Expanding around the clockwork scalar VEV, the relevant Lagrangian terms are

$$
\begin{aligned}
\mathcal{L}_{\mathrm{br}}= & -y_{1} e^{i \phi / f} L H N-y_{2} e^{i \phi / f} L^{c} H^{\dagger} N \\
& -m_{L} L L^{c}-m_{D} N N^{c}-\frac{m_{N^{c}}}{2} N^{c} N^{c}+\text { H.c. }
\end{aligned}
$$

where $\sqrt{2} y_{1}=Y_{1} f / \Lambda_{\mathrm{FN}}$ and $\sqrt{2} y_{2}=Y_{2} f / \Lambda_{\mathrm{FN}}$.

After integrating out the leptons at one loop, a backreaction potential for the relaxion is generated

$$
V_{\mathrm{br}} \sim \frac{y_{1} y_{2} m_{L} m_{D}^{2} m_{N^{c}}}{(4 \pi M)^{2}} H^{\dagger} H \cos \left(\frac{\phi}{f}\right)
$$

where $M \approx \max \left\{m_{L}, m_{N}, m_{N^{c}}\right\}$. The demand for $M_{\mathrm{br}}<4 \pi v$ is then translated to a bound on the masses $m_{L}, m_{N}$, $m_{N^{c}}<4 \pi v$.

As was discussed in Ref. [5], the contribution to the strong $C P$ phase, generated by this sequestered sector, arises at least at two-loop order, and is further suppressed by $\sim \Lambda_{\mathrm{br}}^{4} / v^{4}$. Furthermore, for a sub-GeV relaxion mass, as in our construction, the contribution to the neutron electric dipole moment is below near-future sensitivity [5].

\section{APPENDIX C: MORE DETAILS ON THE PHENOMENOLOGY}

Through the backreaction sector, a relaxion-Higgs mixing is generated. The mixing angle controls the relaxion phenomenology. Within our range of parameters, the relaxion is always long lived on detector scales and can be probed in different processes which we now describe in turn.
A relevant constraint arises from the flavor-violating invisible decay of the kaon. This is bounded at the $90 \%$ C.L. to be $\operatorname{BR}\left(K^{+} \rightarrow \pi+\phi\right)<7.3 \times 10^{-11}$ by combined data from the E787 and E949 experiments [40]. Astrophysical constraints arise from energy loss arguments for the SN1987A supernova. As first proposed in Ref. [36], we require the cooling rate into the relaxion to be less than $6 \times 10^{55} \mathrm{GeV} / \mathrm{s}$. We neglect possible uncertainties coming from the modeling of the neutrino emission from the collapse (see Refs. [62-64]). This bound suffers also from large systematical uncertainties due to the poorly known parameters of the supernova, like its temperature, its core radius, and the neutron density (we fix them, following Ref. [65], to $T=60 \mathrm{MeV}, R_{\text {core }}=10 \mathrm{~km}$, and $\rho_{n}=3 \times 10^{14} \mathrm{~g} / \mathrm{cm}^{3}$ ). A more recent analysis [39] addressed these uncertainties, strengthening the robustness of this bound.

Further bounds can be derived from the cooling rate of the Sun, horizontal branch stars, and red giants $[38,66]$. The late decays of the relaxion can also affect the diffuse extragalactic background light, first computed in Ref. [5]. All these bounds together exclude already all the regions where $\sin \theta \gtrsim 10^{-10}$. Present fifth-force experiments [67-71] do not have the sensitivity to probe a sub-eV mediation with mixing with the SM Higgs smaller than $10^{-15}$.

We finally present, for completeness, the allowed parameter space for the NB relaxion when the Wilson coefficients of the higher-dimensional operators in Eq. (A8) do not satisfy

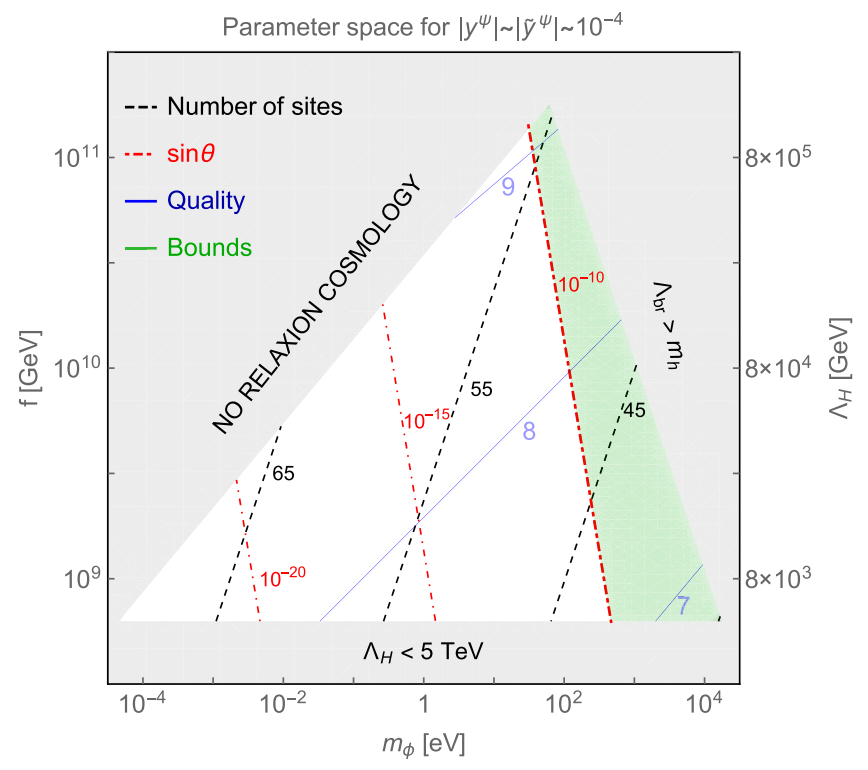

FIG. 2. The white region shows the allowed parameter space of the Nelson-Barr relaxion. The different contour lines indicate the number of clockwork sites (black dashed), the relaxion-Higgs mixing (red dot-dashed), and the minimal dimension of the Planck-suppressed operators that should be forbidden (thin blue). The green-shaded region combines constraints from astrophysics [36-39], extragalactic background light [5], and flavor-violating kaon decays [40]. 
MFV, but have quasidiagonal texture. The upper bound on the spurions $y_{i}^{\psi}$ and $\tilde{y}_{j}^{\psi}$ is 2 orders of magnitude stronger in this case, resulting in a higher decay constant,

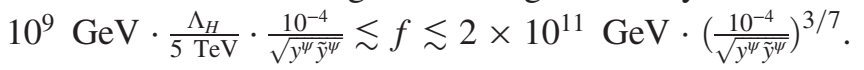

The mass range of the NB relaxion is then $2 \times 10^{-4} \mathrm{eV}$. $\left(\frac{\Lambda_{H}}{5 \mathrm{TeV}}\right)^{5 / 2} \cdot \frac{10^{-4}}{\sqrt{y^{\psi} \tilde{y}^{\Psi}}} \lesssim m_{\phi} \lesssim 0.2 \mathrm{MeV} \cdot \frac{5 \mathrm{TeV}}{\Lambda_{H}} \cdot \frac{\sqrt{y^{\psi} \tilde{y}^{\psi}}}{10^{-4}}$. The available parameter space is presented in Fig. 2.
[1] P. W. Graham, D. E. Kaplan, and S. Rajendran, Phys. Rev. Lett. 115, 221801 (2015).

[2] A. E. Nelson, Phys. Lett. 136B, 387 (1984).

[3] S. M. Barr, Phys. Rev. Lett. 53, 329 (1984).

[4] S. M. Barr, Phys. Rev. D 30, 1805 (1984).

[5] T. Flacke, C. Frugiuele, E. Fuchs, R. S. Gupta, and G. Perez, J. High Energy Phys. 06 (2017) 050.

[6] L. Bento, G. C. Branco, and P. A. Parada, Phys. Lett. B 267, 95 (1991).

[7] M. Dine and P. Draper, J. High Energy Phys. 08 (2015) 132.

[8] J. M. Pendlebury et al., Phys. Rev. D 92, 092003 (2015).

[9] J. Polchinski and M. B. Wise, Phys. Lett. 125B, 393 (1983).

[10] W. Buchmuller and D. Wyler, Phys. Lett. 121B, 321 (1983); 121B, 277(E) (1982).

[11] M. Dugan, B. Grinstein, and L. J. Hall, Nucl. Phys. B255, 413 (1985).

[12] K. Agashe, G. Perez, and A. Soni, Phys. Rev. Lett. 93, 201804 (2004).

[13] R. S. Gupta, Z. Komargodski, G. Perez, and L. Ubaldi, J. High Energy Phys. 02 (2016) 166.

[14] J. R. Espinosa, C. Grojean, G. Panico, A. Pomarol, O. Pujolas, and G. Servant, Phys. Rev. Lett. 115, 251803 (2015).

[15] K. Choi, H. Kim, and S. Yun, Phys. Rev. D 90, 023545 (2014).

[16] K. Choi and S. H. Im, J. High Energy Phys. 01 (2016) 149.

[17] D. E. Kaplan and R. Rattazzi, Phys. Rev. D 93, 085007 (2016).

[18] This bound can be circumvented if other sources of energy dissipation become dominant [19-21].

[19] A. Hook and G. Marques-Tavares, J. High Energy Phys. 12 (2016) 101.

[20] W. Tangarife, K. Tobioka, L. Ubaldi, and T. Volansky, arXiv: 1706.00438.

[21] W. Tangarife, K. Tobioka, L. Ubaldi, and T. Volansky, J. High Energy Phys. 02 (2018) 084.

[22] A. Nelson and C. Prescod-Weinstein, Phys. Rev. D 96, 113007 (2017).

[23] K. S. Jeong and C. S. Shin, J. High Energy Phys. 01 (2018) 121.

[24] This includes also the setup presented in Ref. [19], where the EW scale is selected by the particle production mechanism, and there is no need for a backreaction sector at all.

[25] O. Davidi, R. S. Gupta, G. Perez, D. Redigolo, and A. Shalit, J. High Energy Phys. 08 (2018) 153.

[26] G. D’Ambrosio, G. F. Giudice, G. Isidori, and A. Strumia, Nucl. Phys. B645, 155 (2002).

[27] A. L. Kagan, G. Perez, T. Volansky, and J. Zupan, Phys. Rev. D 80, 076002 (2009).
[28] M. Knig, M. Neubert, and D. M. Straub, Eur. Phys. J. C 74, 2945 (2014).

[29] B. Batell, G. F. Giudice, and M. McCullough, J. High Energy Phys. 12 (2015) 162.

[30] B. Batell, M. A. Fedderke, and L.-T. Wang, J. High Energy Phys. 12 (2017) 139.

[31] J. L. Evans, T. Gherghetta, N. Nagata, and M. Peloso, Phys. Rev. D 95, 115027 (2017).

[32] M. Dine, R. G. Leigh, and A. Kagan, Phys. Rev. D 48, 2214 (1993).

[33] L. Vecchi, J. High Energy Phys. 04 (2017) 149.

[34] S. Abel, R. S. Gupta, and J. Scholtz, arXiv:1810.05153.

[35] R. S. Gupta, J. Reiness, and M. Spannowsky (to be published).

[36] G. Raffelt and D. Seckel, Phys. Rev. Lett. 60, 1793 (1988).

[37] G. Raffelt, Phys. Rev. D 86, 015001 (2012).

[38] E. Hardy and R. Lasenby, J. High Energy Phys. 02 (2017) 033.

[39] J. H. Chang, R. Essig, and S. D. McDermott, J. High Energy Phys. 09 (2018) 051.

[40] S. Adler et al. (E787 and E949 Collaborations), Phys. Rev. D 77, 052003 (2008).

[41] G. Perez and T. Volansky, Phys. Rev. D 72, 103522 (2005).

[42] M. Kamionkowski and J. March-Russell, Phys. Lett. B 282, 137 (1992).

[43] T. Banks and L. J. Dixon, Nucl. Phys. B307, 93 (1988).

[44] L. F. Abbott and M. B. Wise, Nucl. Phys. B325, 687 (1989).

[45] S. R. Coleman and K.-M. Lee, Nucl. Phys. B329, 387 (1990).

[46] R. Kallosh, A. D. Linde, D. A. Linde, and L. Susskind, Phys. Rev. D 52, 912 (1995).

[47] T. Banks and N. Seiberg, Phys. Rev. D 83, 084019 (2011).

[48] T. Higaki, K. S. Jeong, N. Kitajima, and F. Takahashi, J. High Energy Phys. 06 (2016) 150.

[49] We parametrize the breaking by local Planck-suppressed operators and refer to Ref. [46,50] for a discussion of how these are generated from gravitational instantons.

[50] R. Alonso and A. Urbano, arXiv:1706.07415.

[51] R. Coy, M. Frigerio, and M. Ibe, J. High Energy Phys. 10 (2017) 002.

[52] R. F. Dashen, Phys. Rev. D 3, 1879 (1971).

[53] D. Gaiotto, Z. Komargodski, and N. Seiberg, J. High Energy Phys. 01 (2018) 110.

[54] C. D. Froggatt and H. B. Nielsen, Nucl. Phys. B147, 277 (1979).

[55] R. Barbieri, G. Isidori, J. Jones-Perez, P. Lodone, and D. M. Straub, Eur. Phys. J. C 71, 1725 (2011). 
[56] B. Keren-Zur, P. Lodone, M. Nardecchia, D. Pappadopulo, R. Rattazzi, and L. Vecchi, Nucl. Phys. B867, 394 (2013).

[57] F. Sala, Proc. Sci., CORFU2011 (2011) 032.

[58] F. Sala, in Proceedings of the 2nd Workshop on Flavor Symmetries and Consequences in Accelerators and Cosmology, FLASY 12, Dortmund, Germany, 2012 (2012), pp. 247-254.

[59] G. Panico and A. Wulzer, Lect. Notes Phys. 913, 1 (2016).

[60] G. Isidori, Y. Nir, and G. Perez, Annu. Rev. Nucl. Part. Sci. 60, 355 (2010).

[61] O. Gedalia and G. Perez, Physics of the large and the small, TASI 09, in Proceedings of the Theoretical Advanced Study Institute in Elementary Particle Physics, Boulder, Colorado, USA, 2009 (2011), pp. 309-382.

[62] D. Kushnir and B. Katz, Astrophys. J. 811, 97 (2015).

[63] D. Kushnir, arXiv:1502.03111.
[64] K. Blum and D. Kushnir, Astrophys. J. 828, 31 (2016).

[65] N. Ishizuka and M. Yoshimura, Prog. Theor. Phys. 84, 233 (1990).

[66] G. Raffelt, Phys. Rev. D 86, 015001 (2012).

[67] R. Spero, J. K. Hoskins, R. Newman, J. Pellam, and J. Schultz, Phys. Rev. Lett. 44, 1645 (1980).

[68] J. K. Hoskins, R. D. Newman, R. Spero, and J. Schultz, Phys. Rev. D 32, 3084 (1985).

[69] J. Chiaverini, S. J. Smullin, A. A. Geraci, D. M. Weld, and A. Kapitulnik, Phys. Rev. Lett. 90, 151101 (2003).

[70] S. J. Smullin, A. A. Geraci, D. M. Weld, J. Chiaverini, S. P. Holmes, and A. Kapitulnik, Phys. Rev. D 72, 122001 (2005); 72, 129901(E) (2005).

[71] D. J. Kapner, T. S. Cook, E. G. Adelberger, J. H. Gundlach, B. R. Heckel, C. D. Hoyle, and H. E. Swanson, Phys. Rev. Lett. 98, 021101 (2007). 